Artigo

\title{
Caracterização Sinótica e Climatológica de Eventos de Chuva Pós-Frontal no Rio de Janeiro
}

\author{
Suzanna Maria Bonnet ${ }^{1}$ iD, Claudine Pereira Dereczynski ${ }^{1}$, Ana Nunes ${ }^{1}$ \\ ${ }^{1}$ Departamento de Meteorologia, Instituto de Geociências, \\ Universidade Federal do Rio de Janeiro, Rio de Janeiro, RJ, Brasil.
}

Recebido em 24 de Outubro de 2017 - Aceito em 30 de Maio de 2018

\begin{abstract}
Resumo
No Município do Rio de Janeiro (MRJ), verifica-se em certas situações de deslocamento de Sistemas Frontais (SFs), a ocorrência de chuvas fracas a moderadas, mesmo após a completa passagem do SF pelo Estado. Este fenômeno é conhecido na região como precipitação devido ao "efeito de circulação marítima", mas não apresenta documentação, ficando restrito ao conhecimento dos previsores locais. O objetivo deste trabalho é elaborar uma climatologia da frequência de ocorrência desse fenômeno no MRJ para o período 2000-2013 e identificar as condições sinóticas associadas. A partir de imagens de satélite e dados de precipitação, as passagens de SFs pelo MRJ foram classificadas em três tipos: Pós-Frontais Sem Chuva (PFSC); Pós-Frontais Com Chuva (PFCC) e Pós-Frontais Excluídos (PFE). Os resultados indicam que mais da metade dos eventos são do tipo PFE (52\%), 32\% do tipo PFSC e 16\% do tipo PFCC. Os casos PFE predominam no verão, os PFSC no inverno e primavera e os PFCC no outono. Os compostos dos casos PFSC e PFCC utilizando a Reanálise ERA-Interim indicam que a precipitação pós-frontal estaria associada à convergência de fortes ventos de sul junto à costa ao penetrar no continente, formando nuvens estratiformes, apesar do SF não estar mais atuando.
\end{abstract}

Palavras-chave: chuva pós-frontal, circulação marítima, Rio de Janeiro, sistema frontal.

\section{Synoptic and Climatological Characterization of Post-Frontal Rainfall Events in Rio De Janeiro}

\begin{abstract}
In the City of Rio de Janeiro (CRJ), light to moderate rain can occur even after the total displacement of a Frontal System (FS) over the State. This phenomenon is known as precipitation from "sea circulation effect", but there is no documentation about this, being restricted to the knowledge of the local servers. This study aims to characterize this phenomenon climatology (2000-2013), and to identify the associated synoptic conditions. From all the FS passages through CRJ, using satellite imagery and observed precipitation, the events were classified into three categories: No-Rain Post Front (NRPF), Rainy Post Front (RPF) and Excluded Post Front (EPF). The results show that more than half of the events are EPF $(52 \%), 32 \%$ are NRPF and $16 \%$ are RPF. The EPF cases are more frequent in summer, NRPF in winter and spring, and RPF in fall. The composites from NRPF and RPF using ERA-Interim Reanalysis indicate that the post-frontal precipitation is associated with the convergence of strong southerly winds while reaching the continent, leading to the formation of precipitating stratiform clouds, even though the FS is no longer active in the region.
\end{abstract}

Keywords: post-frontal precipitation, seawind circulation, Rio de Janeiro, frontal system.

\section{Introdução}

As frentes frias são os sistemas transientes mais frequentes sobre o continente sul americano (Satyamurty et al., 1998). De acordo com Oliveira (1986), entre as bandas latitudinais de $25^{\circ} \mathrm{S}$ e $20^{\circ} \mathrm{S}$, onde se localiza o Estado do Rio de Janeiro (ERJ), passam uma média de 3,1 a 5,4 Sistemas
Frontais (SFs) por mês, totalizando-se 47,3 SFs por ano, distribuídos mensalmente da seguinte forma: 3,8 (janeiro); 2,5 (fevereiro); 3,1 (março);3,3 (abril); 3,8(maio); 4,4 (junho); 4,1 (julho); 4,2 (agosto); 4,4 (setembro); 5,4 (outubro); 4,3 (novembro) e 4,0 (dezembro).

Autor de correspondência: Suzanna Maria Bonnet, suzannamabonnet@gmail.com. 
De acordo com Satyamurty et al. (1998) e Nobre et al. (1998), a trajetória dos SFs sobre a América do Sul segue dois padrões bastante distintos ao longo do ano. Durante o inverno, as massas de ar frio com alta pressão à superfície e baixas temperaturas, penetram na direção norte-nordeste sobre o continente, no rastro das frentes. Tais massas de ar movem-se sobre o Atlântico e gradualmente perdem sua identidade, misturando-se com o Anticiclone Subtropical do Atlântico Sul (ASAS). A trajetória de verão é mais lenta e caracterizada por maior atividade convectiva devido às complexas interações entre os sistemas transientes de escala sinótica e os sistemas quase estacionários, como a Alta da Bolívia e a Zona de Convergência do Atlântico Sul (ZCAS). As frentes frias no verão penetram rapidamente a partir da Argentina, deslocando-se para nordeste na região da ZCAS, onde podem permanecer estacionárias por um período de 5 a 10 dias, produzindo grandes quantidades de chuva (em torno de $300 \mathrm{~mm}$ em uma semana) nas regiões do Rio de Janeiro, norte e leste de São Paulo, sul e oeste de Minas Gerais, Mato Grosso do Sul e sul e leste do Mato Grosso.

Cavalcanti e Kousky (2009) estudaram as características da passagem de SFs sobre a América do Sul, a partir dos cinquenta eventos de passagens de frentes frias mais intensos ocorridos no período de 1979 a 2005. Seus resultados mostram que quando o SF chega ao sul do Brasil, em baixos níveis predomina escoamento de sudoeste na porção continental da Região Sul do Brasil, e já existe uma marcante convergência de vento sobre a Região Sudeste. Quando o SF atinge a Região Sudeste, os ventos locais adquirem direção sudoeste, inclusive sobre o ERJ. Tal padrão de ventos promove advecção fria e ocasiona queda significativa nas temperaturas em toda Região Sudeste do Brasil.

Além da passagem dos SFs, o posicionamento do ASAS também afeta a precipitação na América do Sul. Lima (1991), utilizando dados de vento na Ilha de Trindade e em Abrolhos, observou que em anos chuvosos no leste da região Nordeste do Brasil (NEB) o ASAS se encontra deslocado para sudeste de sua posição climatológica, promovendo ventos mais intensos e perpendiculares à costa, contribuindo para aumentar o transporte de umidade do oceano para o continente. Por outro lado, de acordo com Lima (1991), nos anos secos o ASAS se encontra afastado do continente, menos ao sul e mais a leste do continente sul americano, em relação à sua posição climatológica. Para o sul do NEB, Chaves e Cavalcanti (2001) afirmam que os períodos secos e chuvosos são afetados pela intensidade e direção do escoamento da Amazônia e também pelo posicionamento da periferia oeste do ASAS. No padrão chuvoso o vento flui da região amazônica para o sul do NEB e o ASAS se encontra deslocado para leste, com os ventos alísios mais fracos sobre a América do Sul. Essa configuração resulta em convergência de umidade sobre o sul do NEB, permitindo o desenvolvimento e organização da
ZCAS ao norte de sua posição normal. No padrão seco o ASAS é deslocado para oeste, intensificando os ventos alísios sobre o Brasil. Nesse caso o vento flui da Amazônia em direção ao sudeste, deslocando a ZCAS para sul. Simulações com um modelo global são elaboradas por Richter et al. (2008) para estudar a dinâmica do ASAS, que de acordo com os autores, é o anticiclone menos estudado do hemisfério sul. Seus resultados confirmam a importância da orografia da América do Sul e da África na localização do ASAS sobre o oceano.

Em geral, após a passagem dos SFs há a atuação do Anticiclone Migratório (AM) na retaguarda de tais sistemas, com temperaturas mais amenas, reduzido teor de umidade e maiores valores de pressão atmosférica, promovendo condições de céu claro ou com poucas nuvens, boa visibilidade e ventos calmos que giram do quadrante sul para norte. Contudo, em determinadas ocasiões, observa-se, que após a passagem dos SFs, o vento continua soprando de quadrante sul, associado ao AM, e continua a chover no município do Rio de Janeiro (MRJ), mesmo quando tal sistema encontra-se afastado do estado, muitas vezes localizado no norte do Espírito Santo ou no sul da Bahia (Domingues e Dereczynski, 2005). Essas chuvas, de intensidade fraca a moderada e de origem estratiforme, são conhecidas como "chuvas por efeito de circulação marítima" (Dereczynski et al., 2009). Tal denominação refere-se à chuva associada à atuação do $\mathrm{AM}$, quando ventos de sul vindos do oceano sopram em direção ao continente.

Estudos sobre chuva pós-frontal são pouco comuns na literatura (Domingues e Dereczynski, 2005; Mello e Dereczynski, 2008; Mello, 2009; Bonnet, 2012), ficando restrito ao conhecimento dos previsores locais, sendo essa, portanto, a principal motivação do presente artigo. Visando auxiliar no entendimento desse tipo de situação, o presente artigo documenta suas principais características, a partir da climatologia de ocorrência e do comportamento sinótico associados. Na Seção 2 encontra-se a descrição dos dados e da metodologia utilizados neste estudo. Na Seção 3, os resultados são apresentados em três subseções: i) A climatologia de passagem de SFs sobre o Rio de Janeiro; ii) A análise dos compostos para os casos PFSC e PFCC e iii) A comparação de dois estudos de casos, um PFSC ocorrido em 14/05/2010 e outro PFCC em 08/04/2010. As conclusões encontram-se na Seção 4.

\section{Dados e Metodologia}

A metodologia para identificação dos casos se desenvolve em 4 passos sequenciais:

1. Inicialmente foi feito um levantamento de todos os casos de passagens de SFs pelo MRJ no período de 2000 a 2013, a partir do Boletim Climanálise do CPTEC/INPE [http://climanalise.cptec.inpe.br/ rclimanl/boletim/].

2. Para cada passagem de SF, foram analisadas imagens dos satélites Geostationary Operational Environmental Satellite (GOES)-10, GOES-12 e GOES-13 no canal 
infravermelho, para determinar a data e o horário de saída de cada sistema do ERJ.

3. Os eventos foram classificados em dois tipos, com base nas imagens de satélite: casos pós-frontais em que foi possível visualizar a saída do SF do ERJ; e casos excluídos, nos quais não foi possível determinar a saída do SF do ERJ. Em detalhe, os casos excluídos (denominados Pós-Frontal Excluído - PFE) foram aqueles em que: (i) o SF se deslocou de forma lenta, promovendo a convecção no interior do continente, ocasionalmente configurando episódios de ZCAS; (ii) casos em que a banda de nebulosidade frontal se deslocou para o oceano após atingir o ERJ, sem atuação no continente e (iii) casos em que o SF se dissipou sobre o ERJ e não se pôde identificar a situação de passagem (ou deslocamento) da frente.

4. Com o objetivo de definir o caráter dos casos pós-frontais, definidos no passo 3 , quanto à ocorrência ou não de chuva, foram utilizados dados observacionais de precipitação das 32 estações pluviométricas do Sistema Alerta Rio. Os dados de precipitação foram acumulados nas $24 \mathrm{~h}$ posteriores à data e horário de saída do SF do ERJ, ou seja, início do período pós-frontal, sendo que esse horário foi arredondado para 00 ou 12 UTC, de acordo com o mais próximo. Caso a precipitação acumulada seja superior a 5 $\mathrm{mm}$ em, no mínimo, 4 estações, o evento é considerado como Pós-Frontal Com Chuva (PFCC) e, caso contrário, o evento é considerado como Pós-Frontal Sem Chuva (PFSC).

A Fig. 1 exemplifica os três tipos de casos de passagens de SFs pelo MRJ (PFSC, PFCC e PFE), a partir de sequências de imagens de satélite.

- PFSC - [linha superior] Nesse caso, o SF atingiu o MRJ em 12/05/2010. Durante o seu deslocamento, o SF provocou chuvas intensas principalmente no leste de Santa Catarina e no nordeste do Rio Grande do Sul, ocasionando inclusive granizo no Paraná. A massa de ar frio que acompanhava o sistema afetou, sobretudo, as Regiões Sul e Sudeste. Após o dia 13/05, o SF, já posicionado na altura do Espírito Santo, deslocou-se para o oceano. Aplicando-se a metodologia descrita acima, o período considerado como pós-frontal foi de 14/05 - 12 UTC a 15/05 - 12 UTC. Não ocorreu precipitação nesse período, sendo, portanto, definido como caso PFSC.

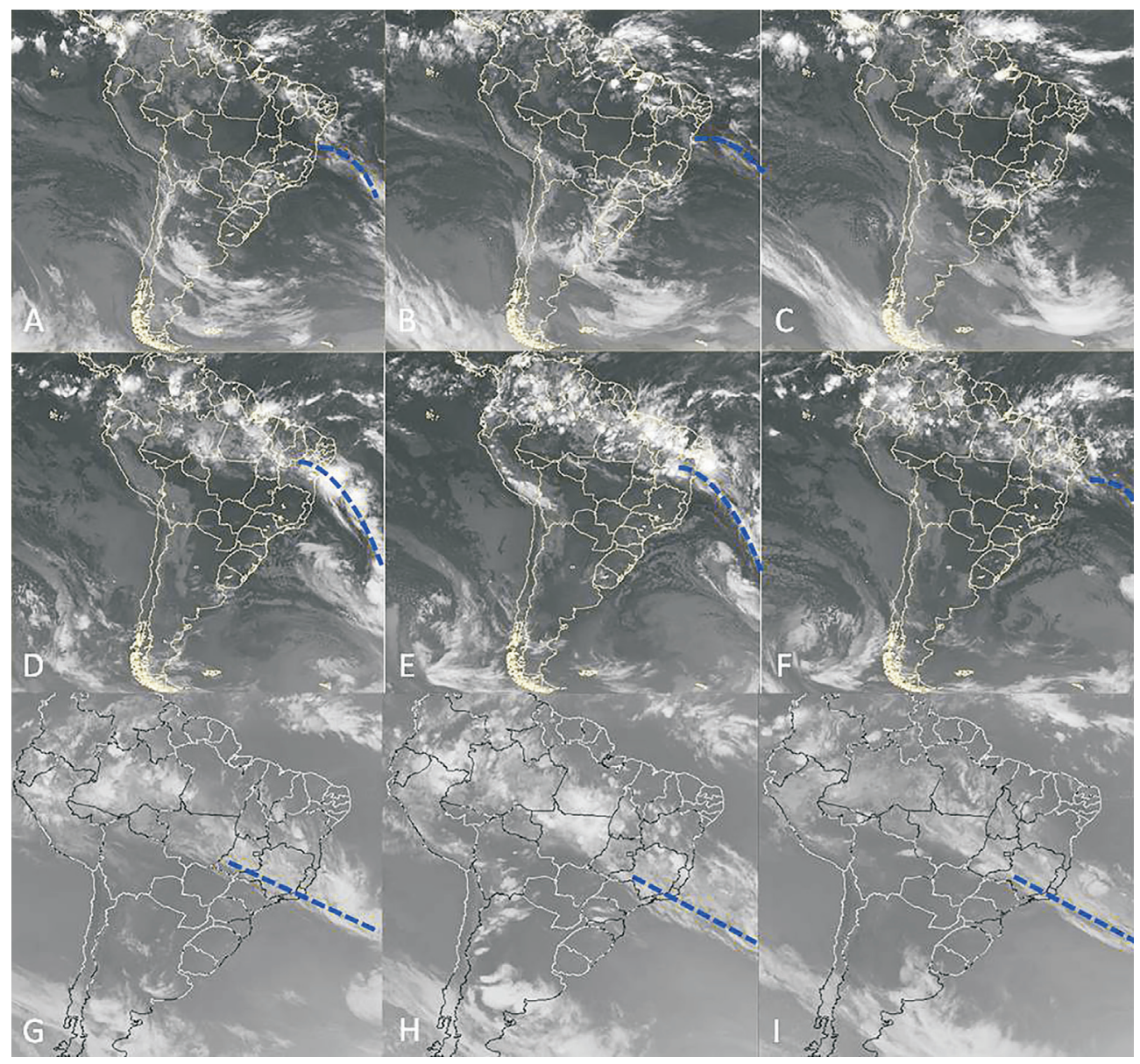

Figura 1 - Três casos de passagens de SF pelo MRJ a partir de sequências de imagens do satélite GOES-12 no canal infravermelho. Caso PFSC (linha superior): (a) 14/05/2010 - 12 UTC, (b) 15/05/2010 - 00 UTC, (c) 15/05/2010 - 12 UTC. Caso PFCC (linha do meio): (d) 08/04/2010 - 12 UTC, (e) 09/04/2010 - 00 UTC, (f) 09/04/2010 - 12 UTC. Caso PFE (linha inferior): (g) 30/11/2004 - 12:09 UTC, (h) 01/12/2004 - 00:09 UTC, (i) 01/12/2004 12:09 UTC. As posições dos SFs encontram-se destacadas em linha tracejada azul. 
- PFCC - [linha do meio] Nesse caso, após a passagem de uma frente fria pelo MRJ, a chuva intensa teve início na noite do dia 05/04/2010 e até as 12 UTC do dia seguinte foram totalizados mais de $100 \mathrm{~mm}$ em todas as estações do Alerta Rio, sendo o maior acumulado em $24 \mathrm{~h}$ de $321 \mathrm{~mm}$, observado no Sumaré. Esse caso está associado ao desastre ocorrido no Morro do Bumba, em Niterói-ERJ. $\mathrm{O}$ evento começou em $05 / 04$, com chuvas extremas sobre a RMRJ provocando deslizamentos de encostas e soterramentos, deixando um total de 257 mortos em todo o Estado. Nos dias subsequentes à passagem do SF, as chuvas continuaram a cair sobre o MRJ, mesmo com a banda de nebulosidade da frente fria já posicionada a norte do Espírito Santo. Aplicando-se a metodologia descrita acima, o período pós-frontal considerado, ou seja, após a saída do SF da região, foi de 08/04 - 12 UTC a 09/04 - 12 UTC. A Fig. 2 apresenta a distribuição da chuva acumulada no período considerado acima, nas estações pluviométricas do Sistema Alerta Rio.

- PFE - [linha inferior] Nesse caso, o SF atingiu o MRJ em 29/11/2004, atuando sobre a região Sudeste ainda nos dias 01 e 02/12/2004, deslocando-se posteriormente para o oceano.

Após a classificação dos casos pós-frontais nas categorias PFSC e PFCC, a fim de se obter os aspectos sinóticos relevantes entre os dois tipos de casos, foram elaborados compostos (campos médios), a partir da Reanálise ERA-Interim (Dee et al., 2011) do European Centre for Medium Range Weather Forecasts (ECMWF), com as seguintes variáveis: pressão atmosférica ao nível médio do mar $(\mathrm{hPa})$, componentes zonal e meridional do vento $\left(\mathrm{m} . \mathrm{s}^{-1}\right)$ a $10 \mathrm{~m}$ de altura, temperatura do $\operatorname{ar}\left({ }^{\circ} \mathrm{C}\right)$ a $2 \mathrm{~m}$ de altura, movimento vertical $\left(\mathrm{Pa}^{-1} \mathrm{~s}^{-1}\right)$ em $1000 \mathrm{hPa}$ e umidade específica $\left(\mathrm{g} \cdot \mathrm{kg}^{-1}\right) \mathrm{em} 1000 \mathrm{hPa}$.

Os dois estudos de casos de PFSC e PFCC, utilizados como exemplo nesta seção, respectivamente, casos de maio/2010 e de abril/2010, são apresentados na Seção 3. Os campos analisados para os estudos de caso, também utilizando a Reanálise ERA-Interim, são: campos de espessura (mgp) da camada entre 1000 e $500 \mathrm{hPa}$; pressão atmosférica ao nível médio do mar (hPa); convergência do vento em $1000 \mathrm{hPa}\left(\mathrm{s}^{-1}\right)$; fluxo de umidade verticalmente integrado $\left(\mathrm{kg} \cdot \mathrm{m}^{-1} \mathrm{~s}^{-1}\right)$ entre 1000 e $300 \mathrm{hPa}$; água precipitável $(\mathrm{mm})$. Ademais, perfis verticais são construídos, sobre um ponto localizado no município do Rio de janeiro $\left(22,5^{\circ}\right.$ $\left.\mathrm{S} / 43,5^{\circ} \mathrm{W}\right)$, exibindo valores de movimento vertical $\left(\mathrm{hPa} \cdot \mathrm{s}^{-1}\right)$, umidade específica $\left(\mathrm{g} \cdot \mathrm{kg}^{-1}\right)$, e direção e intensidade do vento horizontal entre os níveis de 1000 e $300 \mathrm{hPa}$.

\section{Resultados}

\subsection{Climatologia de passagem de sistemas frontais sobre o Rio de Janeiro}

O levantamento de todos os casos de passagem de SFs identificados pelo Boletim Climanálise do CPTEC/INPE pelo MRJ durante o período de 2000 a 2013

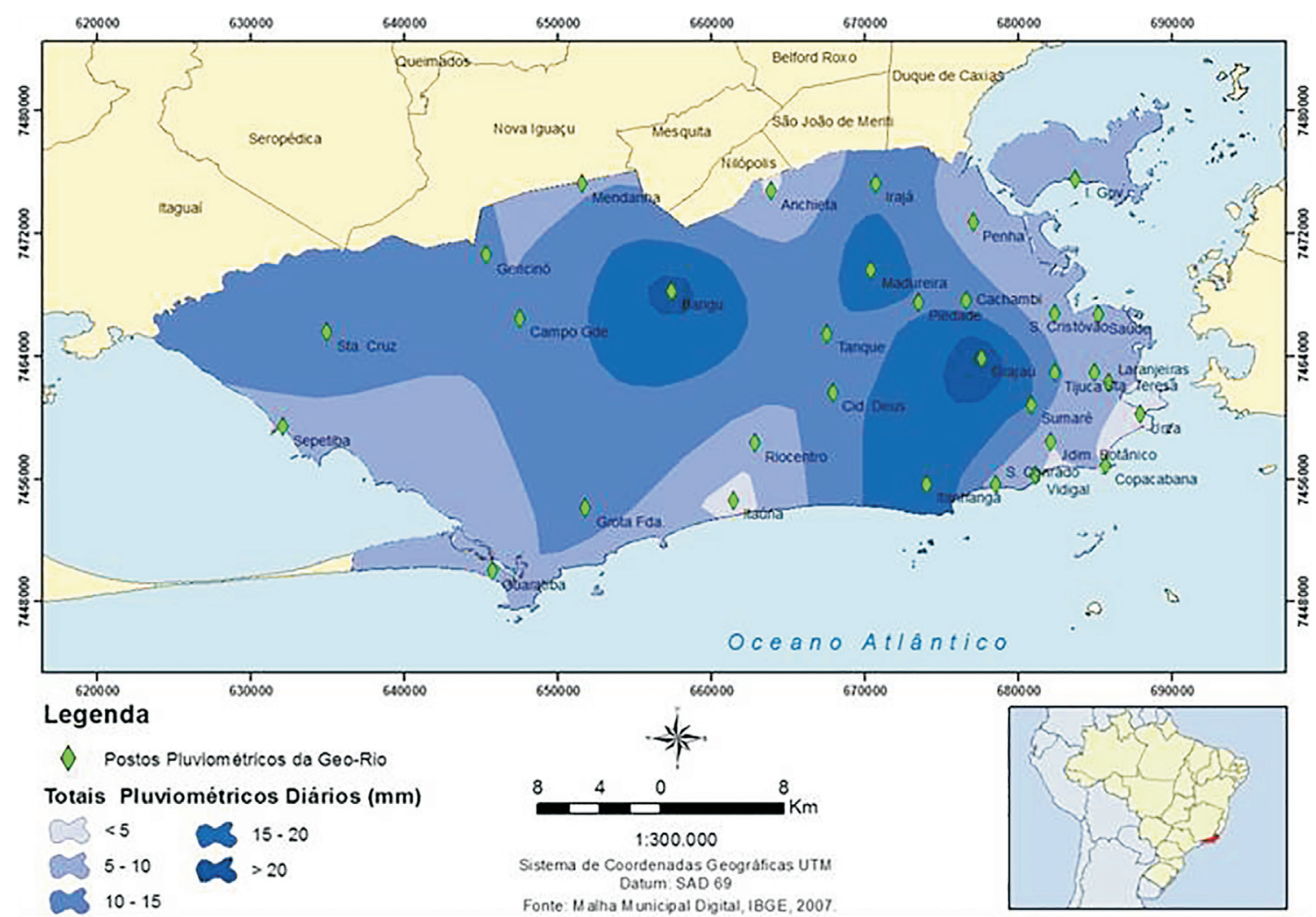

Figura 2 - Distribuição espacial da chuva acumulada no período de 08/04 - 12 UTC a 09/04 - 12 UTC (exemplo PFCC). 
(14 anos) totalizou 473 eventos, ou seja, uma média de 33,8 eventos por ano. Tal valor é ligeiramente inferior ao encontrado por Oliveira (1986) para toda a faixa $25^{\circ} \mathrm{S}-20^{\circ} \mathrm{S}$, que obteve uma média de 47,3 eventos por ano. A separação dos eventos no MRJ por categoria, como definido na metodologia, resultou em: 150 PFSC (sem chuva), 75 PFCC (com chuva) e 248 PFE (excluídos). A Fig. 3 e a Tabela 1 apresentam a distribuição mensal desses eventos.

Considerando-se o número total de SFs, ou seja, a soma dos casos PFSC, PFCC e PFE, observa-se que a frequência de ocorrência de SFs é maior entre maio e outubro, oscilando entre 3,1 e 3,6. Essa média é fortemente reduzida a partir de novembro, com o estabelecimento, do Sistema de Monção da América do Sul em meados de outubro (Gan et al., 2004) atingindo 1,6 sistemas em fevereiro. Nesses meses mais quentes, como mencionado anteriormente, os SFs frequentemente se associam à convecção do interior do continente ao atingirem a região em estudo, permanecendo estacionários ou evoluindo em zonas de convergência. Em comparação com Oliveira (1986) que quantificou a passagem de SFs pela banda latitudinal entre 25 e $20^{\circ} \mathrm{S}$, os valores médios mensais encontrados neste trabalho, que considerou apenas os SFs que atingiram o MRJ, são ligeiramente inferiores. Apesar dessas diferenças, pode se notar uma tendência de aumento do número de SFs do final do verão até o início da primavera em ambas as climatologias.

Mais da metade dos SFs que atingem o ERJ (aproximadamente $52 \%$ ) não evoluem de forma clássica, sendo classificados como PFE. Dos SFs restantes, 32\% são PFSC e 16\% são PFCC. Ressalta-se da Tabela 1 que os casos PFCC ocorrem com maior frequência no outono, sendo maio e julho os meses de maior frequência de ocorrência (média de 0,9 sistema por mês). Durante todo o período estudado, não houve ocorrência de caso PFCC no mês de dezembro e em janeiro a média atinge apenas 0,1 caso. Os casos PFSC predominam no inverno e primavera, com máximos nos meses de agosto e setembro (médias de 1,4 e 1,5 sistemas, respectivamente). Nesses meses, a massa de ar na retaguarda do SF apresenta-se mais seca e mais fria, o que promove o aumento da pressão atmosférica no centro

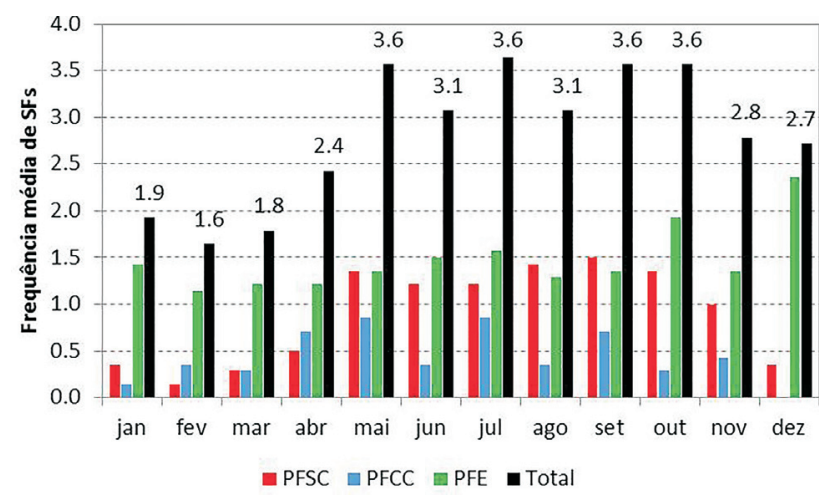

Figura 3 - Frequência média mensal de eventos PFCC, PFSC, PFE e Total, conforme dados contidos na Tabela 1. do AM, inibindo a convecção sobre o continente. No verão verifica-se um declínio nos casos PFSC, com mínimo de 0,1 caso em fevereiro. Nota-se que nos meses de fevereiro e abril os eventos PFCC são mais frequentes do que os eventos PFSC, apesar da baixa frequência de ambos, e no mês de março esses ocorrem com a mesma frequência. Por outro lado, no mês de outubro a frequência de casos PFSC é 4,8 vezes maior do que os casos PFCC. Os casos PFE, como esperado, predominam no verão. Com relação à distribuição mensal dos casos PFE, nota-se uma concentração maior em dezembro (2,4 em média) e outubro (1,9 em média). Verifica-se também, a partir dos dados apresentados na Tabela 1, uma grande variabilidade interanual na frequência de SFs, como a ocorrência de apenas 15 sistemas em 2012 e de $56 \mathrm{em} 2000$. No ano de 2012, os poucos e fracos eventos de ZCAS configurados (Climanálise, 2012) e o reduzido número de SFs, promoveu anomalias negativas de precipitação na maior parte da Região Sudeste do Brasil, nos meses de fevereiro, março, abril, julho, agosto, setembro, outubro e dezembro.

\subsection{Análise dos compostos}

O composto PFSC refere-se ao campo médio dos 150 casos PFSC, selecionados anteriormente. Já o composto PFCC refere-se ao campo médio dos 75 casos PFCC.

A Fig. 4 apresenta os compostos dos campos de pressão atmosférica ao nível médio do mar e divergência dos ventos a $10 \mathrm{~m}$ de altura para os casos PFSC (Fig. 4 a) e PFCC (Fig. 4 b). Notam-se nesses campos duas situações sinóticas distintas: no composto PFCC o cavado sobre o oceano, na retaguarda do SF, encontra-se mais amplificado e o anticiclone migratório (AM) se encontra mais intensificado do que no composto PFSC. Na Fig. 4 b (Fig. 4 a) observa-se que o AM apresenta centro de 1022 (1020) hPa sobre uma área extensa (reduzida) e o cavado frontal encontra-se mais (menos) pronunciado. A convergência associada ao SF em PFCC também é mais intensa (inferior a $-12 \times 10^{-6} \mathrm{~s}^{-1}$ ) em toda a sua extensão, do que em PFSC (entre $\left.-10 \mathrm{e}-12 \times 10^{-6} \mathrm{~s}^{-1}\right)$. Sobre o ERJ a convergência junto à costa é mais intensa em PFCC, o que concorda com o cavado observado na borda nordeste do AM (Fig. 4 b).

A Fig. 5 apresenta os compostos de linhas de corrente e movimento vertical (ômega), ambos em $1000 \mathrm{hPa}$, para os casos PFSC (Fig. 5 a) e PFCC (Fig. 5 b). Nota-se que, ao sul do ERJ, os ventos provenientes do AM sopram de sul e com giro anticiclônico nos dois casos, perpendiculares à linha da costa. Com relação ao campo de ômega, em ambos os casos os valores são negativos (movimento ascendente) sobre o ERJ, sendo mais intensos no composto PFCC, atingindo valores menores que $-10 \mathrm{hPa} . \mathrm{s}^{-1}$, do que no composto PF$\mathrm{SC}$, em que os valores oscilam em torno de $-3 \mathrm{a}-10 \mathrm{hPa} \cdot \mathrm{s}^{-1}$.

Os compostos de temperatura do ar a $2 \mathrm{~m}$ de altura e direção e intensidade do vento a $10 \mathrm{~m}$ de altura são apresentados na Fig. 6. Como esperado, os ventos de sul provenientes do AM são mais intensos no composto PFCC do 
Tabela 1 - Frequência de casos tipo PFSC, PFCC e PFE no período de janeiro de 2000 a dezembro de 2013.

\begin{tabular}{|c|c|c|c|c|c|c|c|c|c|c|c|c|c|c|}
\hline Ano & Tipo & Jan & $\mathrm{Fev}$ & Mar & Abr & Mai & Jun & Jul & Ago & Set & Out & Nov & Dez & Tot \\
\hline \multirow[t]{3}{*}{2000} & PFSC & 1 & - & 1 & 1 & 1 & 2 & - & 1 & 3 & 1 & 2 & 1 & 14 \\
\hline & PFCC & - & 1 & - & 1 & - & - & 3 & - & - & 1 & - & - & 6 \\
\hline & PFE & 4 & 3 & 4 & 1 & 3 & 3 & 3 & 4 & 1 & 3 & 4 & 3 & 36 \\
\hline \multirow[t]{3}{*}{2001} & PFSC & - & - & - & - & - & 1 & - & - & - & 3 & 1 & 1 & 6 \\
\hline & PFCC & - & - & - & - & 1 & - & 1 & - & 1 & - & - & - & 3 \\
\hline & PFE & 1 & 1 & 2 & 3 & 1 & 3 & 3 & - & 3 & - & 3 & 3 & 23 \\
\hline \multirow[t]{3}{*}{2002} & PFSC & - & - & - & - & 1 & - & 3 & 2 & 1 & 2 & 2 & - & 11 \\
\hline & PFCC & - & 1 & 1 & - & 1 & 1 & - & - & 1 & - & 1 & - & 6 \\
\hline & PFE & 2 & - & - & 1 & 1 & 2 & 2 & 1 & 2 & 1 & 1 & 5 & 18 \\
\hline \multirow[t]{3}{*}{2003} & PFSC & 1 & - & - & 1 & 3 & 2 & - & 1 & 2 & 3 & 1 & - & 14 \\
\hline & PFCC & - & - & 2 & 2 & 2 & - & 1 & 1 & 2 & - & 1 & - & 11 \\
\hline & PFE & 2 & 1 & 2 & 1 & - & 3 & 2 & 2 & 1 & 3 & 3 & 5 & 25 \\
\hline \multirow[t]{3}{*}{2004} & PFSC & - & - & 1 & 1 & 1 & - & - & 1 & 1 & - & - & - & 5 \\
\hline & PFCC & 2 & 1 & - & - & 1 & 1 & 3 & 1 & 1 & 1 & 3 & - & 14 \\
\hline & PFE & - & 3 & 2 & 2 & 5 & 1 & 1 & 3 & 2 & 4 & 1 & 3 & 27 \\
\hline \multirow[t]{3}{*}{2005} & PFSC & 1 & - & - & 1 & 2 & 1 & 1 & 2 & 2 & 2 & 2 & 1 & 15 \\
\hline & PFCC & - & 1 & 1 & - & 1 & - & 1 & - & 2 & - & - & - & 6 \\
\hline & PFE & 2 & 3 & 2 & 3 & 2 & 1 & 2 & 1 & 2 & 4 & 4 & 5 & 31 \\
\hline \multirow[t]{3}{*}{2006} & PFSC & - & 1 & 1 & 2 & 1 & 1 & 2 & 1 & 1 & 1 & 2 & 1 & 14 \\
\hline & PFCC & - & - & - & 2 & 2 & 3 & 1 & 1 & 2 & - & - & - & 11 \\
\hline & PFE & 3 & 2 & 2 & - & - & - & - & - & 1 & 2 & - & 1 & 11 \\
\hline \multirow[t]{3}{*}{2007} & PFSC & - & 1 & - & 1 & 3 & 2 & 1 & - & 1 & 1 & 1 & - & 11 \\
\hline & $\mathrm{PFCC}$ & - & - & - & - & 1 & - & 1 & 1 & - & - & - & - & 3 \\
\hline & PFE & 2 & 1 & 1 & 3 & 1 & 2 & 4 & 3 & 2 & 2 & 3 & 2 & 26 \\
\hline \multirow[t]{3}{*}{2008} & PFSC & - & - & - & - & 1 & 2 & 2 & 3 & 1 & - & - & 1 & 10 \\
\hline & $\mathrm{PFCC}$ & - & - & - & 1 & - & - & - & - & - & - & - & - & 1 \\
\hline & PFE & 2 & 2 & 1 & 2 & 2 & 1 & & 1 & 2 & 5 & - & 3 & 21 \\
\hline \multirow[t]{3}{*}{2009} & PFSC & 1 & - & - & - & - & 2 & 2 & 3 & 2 & 1 & - & - & 11 \\
\hline & PFCC & - & - & - & - & - & - & - & - & - & - & - & - & 0 \\
\hline & PFE & 1 & - & - & - & 2 & 1 & 2 & - & 2 & 1 & - & 2 & 11 \\
\hline \multirow[t]{3}{*}{2010} & PFSC & - & - & - & - & 2 & 1 & - & - & 1 & 3 & 1 & - & 8 \\
\hline & PFCC & - & 1 & - & 1 & - & - & - & 1 & - & - & - & - & 3 \\
\hline & PFE & - & - & - & 1 & 1 & 1 & 1 & 2 & - & 1 & - & - & 7 \\
\hline \multirow[t]{3}{*}{2011} & PFSC & - & - & - & - & 2 & 3 & 1 & 2 & 2 & - & 1 & - & 11 \\
\hline & PFCC & - & - & - & - & 2 & - & - & - & 1 & 1 & - & - & 4 \\
\hline & PFE & - & - & - & - & - & - & 1 & 1 & & 1 & - & 1 & 4 \\
\hline \multirow[t]{3}{*}{2012} & PFSC & - & - & 1 & & 1 & - & 3 & 1 & 2 & 1 & - & - & 9 \\
\hline & PFCC & - & - & - & 2 & - & - & - & - & - & - & 1 & - & 3 \\
\hline & PFE & 1 & - & - & - & - & 2 & - & - & - & - & - & - & 3 \\
\hline \multirow[t]{3}{*}{2013} & PFSC & 1 & - & - & - & 1 & - & 2 & 3 & 2 & 1 & 1 & - & 11 \\
\hline & PFCC & - & - & - & 1 & 1 & - & 1 & - & - & 1 & - & - & 4 \\
\hline & PFE & - & - & 1 & - & 1 & 1 & 1 & - & 1 & - & - & - & 5 \\
\hline \multirow[t]{3}{*}{ Total mensal } & PFSC & 5 & 2 & 4 & 7 & 19 & 17 & 17 & 20 & 21 & 19 & 14 & 5 & \\
\hline & PFCC & 2 & 5 & 4 & 10 & 12 & 5 & 12 & 5 & 10 & 4 & 6 & 0 & \\
\hline & PFE & 20 & 16 & 17 & 17 & 19 & 21 & 22 & 18 & 19 & 27 & 19 & 33 & \\
\hline
\end{tabular}


(a)

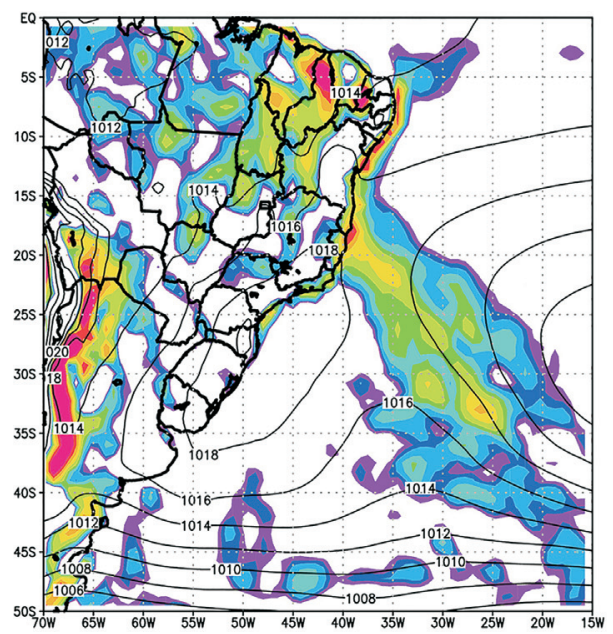

(b)

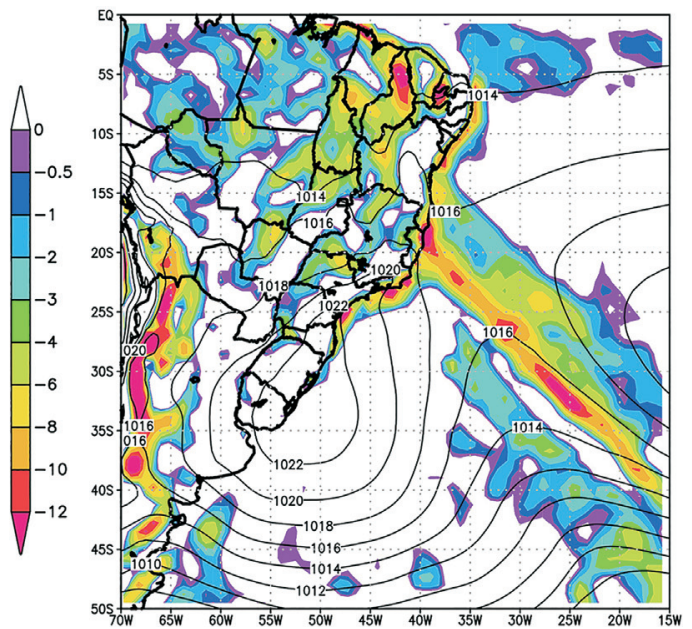

Figura 4 - Compostos de pressão atmosférica ao nível médio do mar (isolinhas a cada $2 \mathrm{hPa}$ ) e valores negativos de divergência do vento $\left(10^{-6} \mathrm{~s}^{-1}\right)$ a $10 \mathrm{~m}$ de altura para: (a) 150 casos PFSC e (b) 75 casos PFCC.

(a)

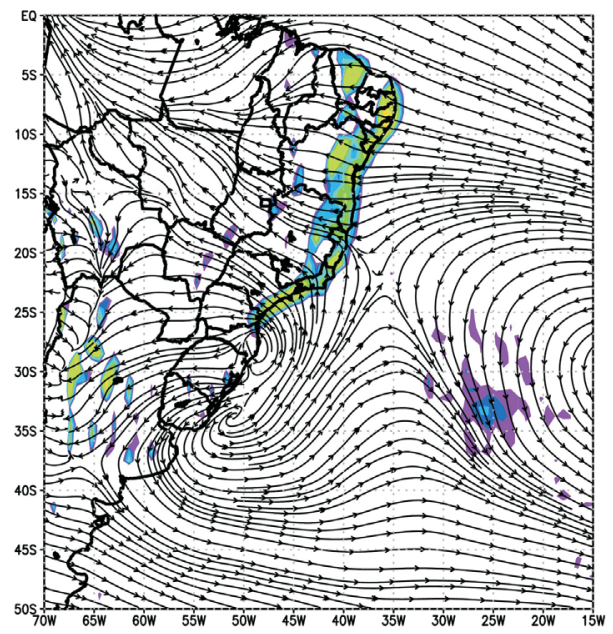

(b)

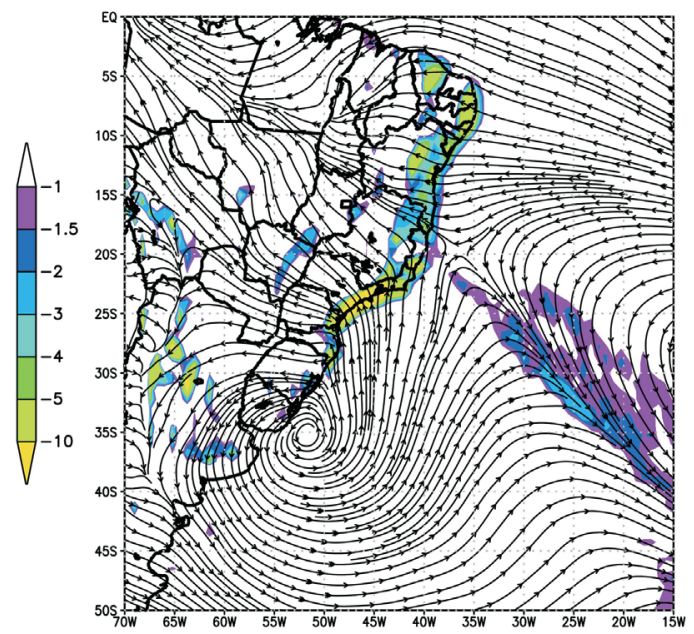

Figura 5 - Compostos de movimento vertical (valores negativos em hPa.s ${ }^{-1}$ ) e linhas de corrente em 1000 hPa para: (a) 150 casos PFSC e (b) 75 casos PFCC.

que no composto PFSC. Ressaltam-se dois gradientes térmicos no campo de temperatura, um localizado no continente sobre a Região Sul do Brasil e outro no oceano na costa dos Estados do Rio de Janeiro e de São Paulo. O gradiente térmico sobre a Região Sul é mais intenso no caso PFCC, devido às menores temperaturas no centro do AM. Ainda no composto PFCC, a interação entre o gradiente térmico sobre o oceano, destacado anteriormente, e os ventos mais intensos sugere a ocorrência de advecção quente mais eficiente em PFCC do que em PFSC.

\subsection{Comparação de dois estudos de casos}

Nesta subseção é feita uma comparação entre os dois casos utilizados como exemplos de PFSC e PFCC na Seção 2, ou seja, o caso PFSC ocorrido em 14/05/2010 e o caso PFCC de 08/04/2010. O objetivo é destacar algumas di- ferenças na situação sinótica favorável a ocorrência de precipitação nas situações pós-frontais.

Na Fig. 1 são mostradas imagens do satélite GOES12 no canal IV para os dois casos. Observa-se, na Fig. 1 a, para o caso PFSC, o posicionamento do SF sobre o Oceano no dia 14/05 - 12 UTC na altura do centro da Bahia e sem nebulosidade sobre o Rio de Janeiro. Na Fig. 1 d, referente ao caso PFCC, em 08/04 - 12 UTC, o SF encontra-se posicionado no norte da Bahia associado a um ciclone extratropical sobre o oceano, e promovendo a formação de nebulosidade convectiva. A precipitação no caso PFCC, acumulada entre os dias 08/04 - 12 UTC e 09/04 - 12 UTC (Fig. 2) apresentou máximos em torno de 20 a $30 \mathrm{~mm} / \mathrm{dia}$, nas Zonas Norte e Oeste da cidade. Em outros dois estudos de caso PFCC, realizados por Domingues e Dereczynski (2005) e Mello (2009), para os dias 21/02/2005 e 
(a)

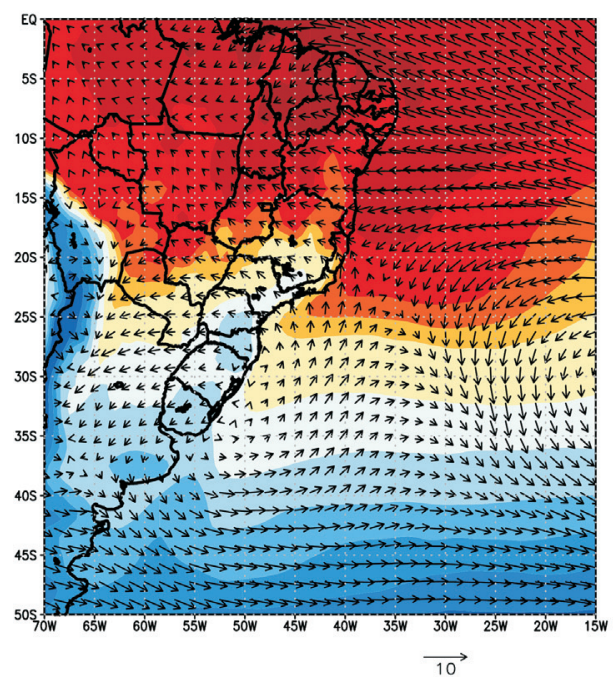

(b)

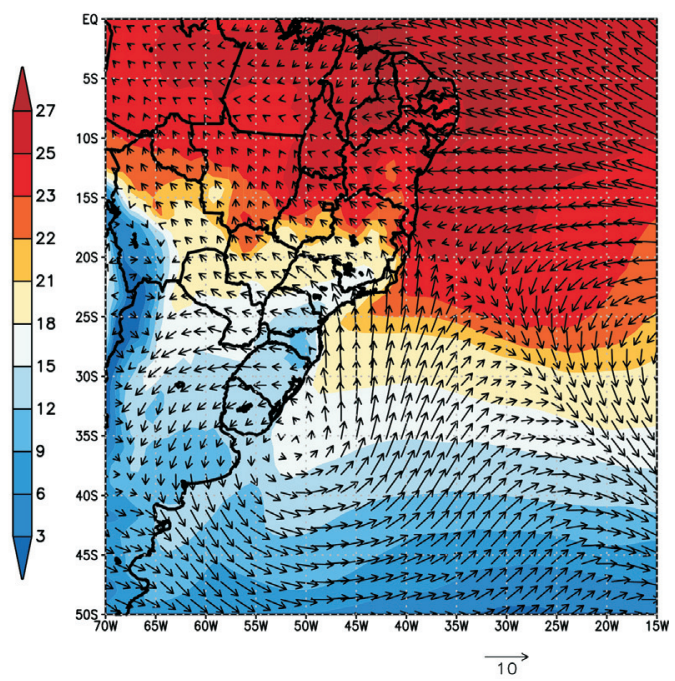

Figura 6 - Compostos de temperatura do ar a $2 \mathrm{~m}$ de altura e vento $\left(\mathrm{m} \cdot \mathrm{s}^{-1}\right)$ a $10 \mathrm{~m}$ de altura para: (a) 150 casos PFSC e (b) 75 casos PFCC.

07/09/2006, respectivamente, os máximos de chuva concentraram-se próximo ao litoral.

Para os estudos de caso, foram elaborados os mesmos campos apresentados na climatologia (figuras não apresentadas). Assim como observado na climatologia, no caso PFCC, ocorreu maior convergência em superfície em comparação ao caso PFSC, sendo que o gradiente de pressão foi maior no caso PFCC, provocando ventos mais intensos. $\mathrm{O}$ movimento ascendente foi mais intenso no caso PFCC do que no caso PFSC, provavelmente devido aos fortes ventos perpendiculares à costa, o que está em conformidade com a climatologia. Com relação à advecção térmica na região, no caso PFCC foi positiva sobre o litoral do Rio de Janeiro, já no caso PFSC a advecção térmica foi nula.

A Fig. 7 apresenta os campos de água precipitável entre 1000 e $300 \mathrm{hPa}$; pressão atmosférica ao nível médio do mar e convergência do vento em $1000 \mathrm{hPa}$, para os casos PFSC e PFCC. Nota-se diferenças significativas entre os

(a)

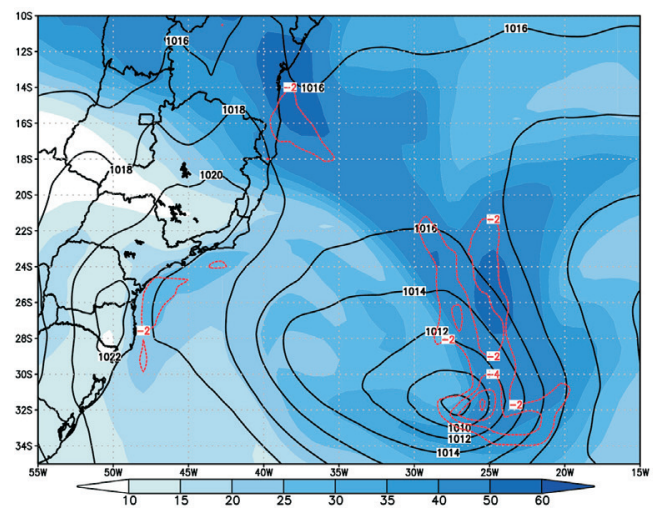

dois casos. No caso PFCC (Fig. 7 b) o centro de baixa pressão de $992 \mathrm{hPa}$, associado ao SF, localizado no sul da Bahia, é mais intenso do que no caso PFSC (Fig. 7 a), com núcleo de $1008 \mathrm{hPa}$. A comparação entre os campos de água precipitável para ambos os casos também indica uma diferença marcante, com valores mais elevados coincidindo com áreas de maior convergência dos ventos, em ambos os casos, porém apresentado valores mais elevados no caso PFCC do que no caso PFSC. Sobre o ERJ os valores de água precipitável oscilam entre 15 e 20 mm (20 e $25 \mathrm{~mm}$ ) no caso PFSC (no caso PFCC).

Na Fig. 8 estão apresentados os campos de espessura entre 1000 e $500 \mathrm{hPa}$, movimento vertical (ômega) em 500 $\mathrm{hPa}$ e linhas de corrente em $500 \mathrm{hPa}$. No caso PFCC (Fig. 8 b) as linhas de mesma espessura exibem um cavado frio pronunciado sobre o Oceano Atlântico, a leste das Regiões Sul e Sudeste do Brasil e uma crista quente a leste do cavado, gerando um forte gradiente de temperatura na

(b)

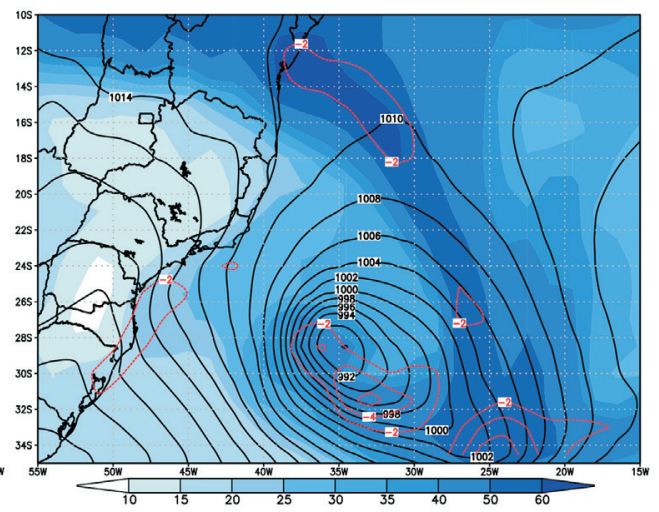

Figura 7 - Água precipitável $(\mathrm{mm})$ da camada 1000-300 hPa (em cores), pressão atmosférica ao nível médio do mar (linhas cheias a cada $2 \mathrm{hPa}$ ) e convergência do vento em $1000 \mathrm{hPa}$ (valores negativos a cada $-2 \times 10^{-6} \mathrm{~s}^{-1}$ ) para: (a) caso PFSC em 14/05/2010 - 12 UTC e (b) caso PFCC em 08/04/2010 12 UTC. 
região de estudo. O vórtice ciclônico localizado a aproximadamente $28^{\circ} \mathrm{S} / 35^{\circ} \mathrm{O}$, apresenta núcleo relativamente quente desde superfície até $500 \mathrm{hPa}$, e se inclina em direção ao ar frio em $200 \mathrm{hPa}$ (figura não apresentada). No caso PFSC (Fig. 8 a), a distribuição horizontal das linhas de espessura exibe um gradiente térmico mais fraco. No campo de linhas de corrente em $500 \mathrm{hPa}$ (Fig. 8 a), existe um cavado e não um vórtice fechado como no caso PFCC.

Na Fig. 9 estão os campos do fluxo de umidade verticalmente integrado entre 1000 e $300 \mathrm{hPa}$ para os casos PFSC e PFCC. Observa-se mais uma vez que no caso PFCC (Fig. 9 a) o fluxo é mais intenso do que no caso PFSC, já que os ventos são mais intensos e que o conteúdo de umidade também é maior do que no caso PFSC (Fig. 9 a). No ERJ os valores dessa variável oscilam entre $100 \mathrm{e}$ $150 \mathrm{~kg} \cdot \mathrm{m}^{-1} \mathrm{~s}^{-1}$ no caso PFSC e entre 300 e $400 \mathrm{~kg} \cdot \mathrm{m}^{-1} \mathrm{~s}^{-1}$ no caso PFCC.

A Fig. 10 apresenta a evolução temporal dos perfis verticais entre os níveis de 1000 e $300 \mathrm{hPa}$ de movimento vertical, umidade específica, e direção e intensidade do vento horizontal, sobre um ponto localizado no município do Rio de janeiro $\left(22,5^{\circ} \mathrm{S} / 43,5^{\circ} \mathrm{W}\right)$, para ambos os casos. No caso PFSC nota-se a passagem do SF no dia 12/05, com intensificação do movimento ascendente, contudo, durante

(a)

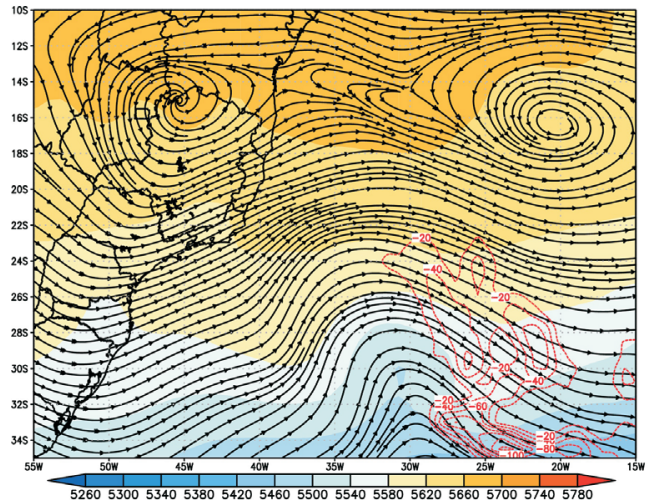

a passagem da frente e no período pós-frontal, desde a superfície até $800 \mathrm{hPa}$, o movimento foi predominantemente subsidente fraco. No caso PFCC, nota-se inicialmente a passagem do SF no dia 06/04, com intensificação do movimento ascendente. Durante o período pós-frontal analisado (entre 08 e 09/04), continuou predominando movimento ascendente desde a superfície até $850 \mathrm{hPa}$.

No perfil de umidade específica pode-se notar que durante o período analisado no caso PFSC, a atmosfera encontrava-se seca como um todo (isolinhas próximas, intenso gradiente vertical de umidade específica), com valores entre $10 \mathrm{e} 11 \mathrm{~g} \cdot \mathrm{kg}^{-1}$ próximos à superfície. Já o caso PFCC não apresenta um gradiente de umidade tão intenso, ou seja, a atmosfera encontra-se mais úmida e não há variação da umidade à superfície, ficando essa em torno de 10 a 12 g. $\mathrm{kg}^{-1}$.

No caso PFSC, observa-se a fraca intensidade dos ventos em baixos níveis (superfície até $850 \mathrm{hPa}$ ) durante e após a passagem do SF. A componente oeste do vento se estende por toda a camada ao longo do período estudado. Contrariamente, o caso PFCC apresenta ventos de quadrante sul desde a superfície até o nível de $500 \mathrm{hPa}$ e com intensidade elevada após a passagem do SF, o giro do vento

(b)

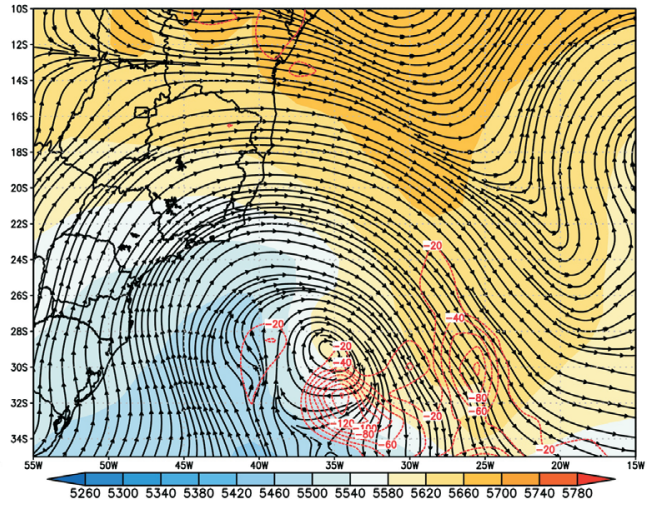

Figura 8 - Espessura (mgp) da camada 1000-500 hPa (em cores), linhas de corrente em 500 hPa e movimento vertical (valores negativos em hPa.s ${ }^{-1}$; linha pontilhada em vermelho) em 500 hPa para: (a) caso PFSC em 14/05/2010 - 12 UTC e (b) caso PFCC em 08/04/2010 - 12 UTC.

(a)

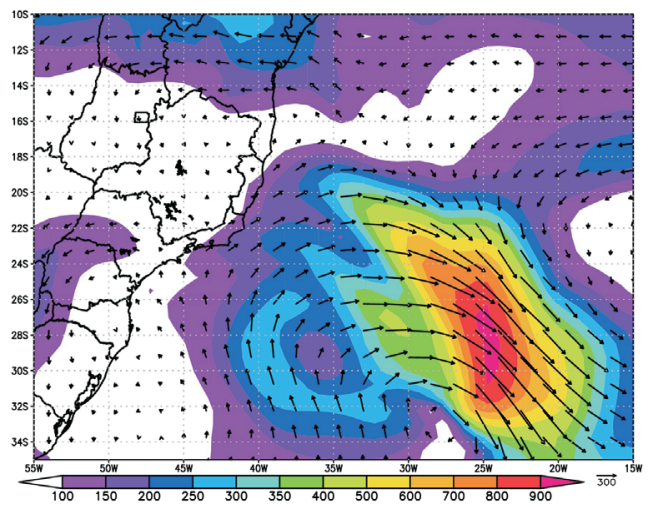

(b)

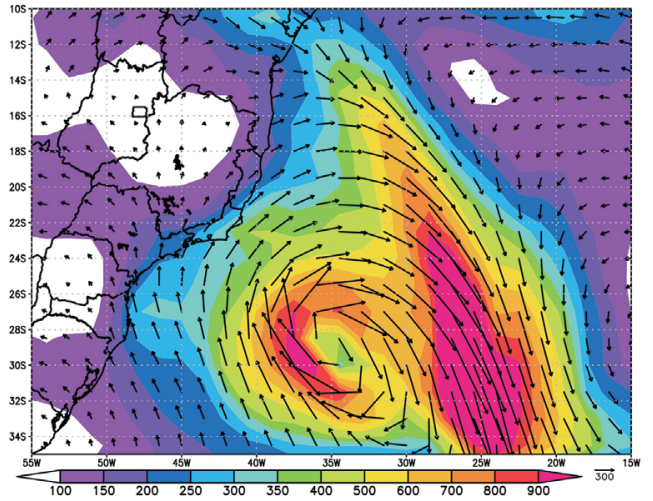

Figura 9 - Fluxo de umidade verticalmente integrado $\left(\mathrm{kg} \cdot \mathrm{m}^{-1} \mathrm{~s}^{-1}\right)$ entre 1000 e $300 \mathrm{hPa}$ para: (a) caso PFSC em 14/05/2010 - 12 UTC e (b) caso PFCC em 08/04/2010 - 12 UTC. 
(a)

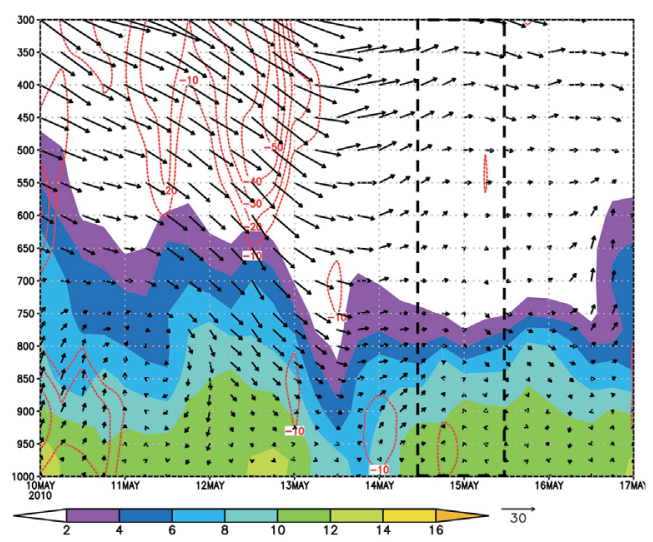

(b)

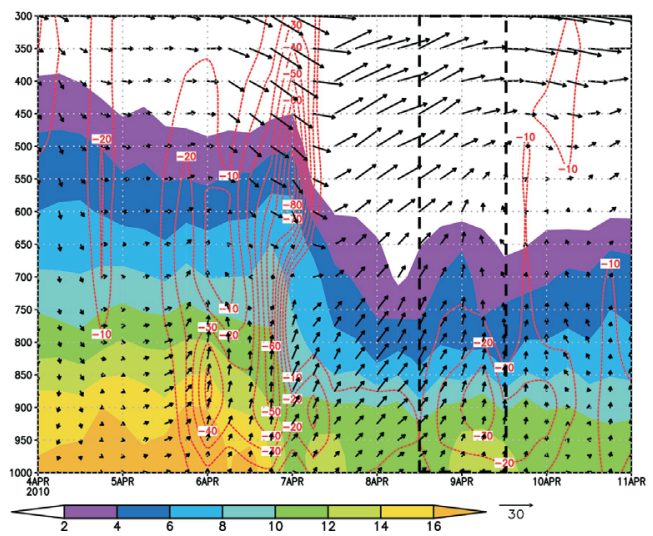

Figura 10 - Evolução temporal dos perfis verticais de movimento vertical (linha vermelha pontilhada a cada $-10 \mathrm{hPa} \cdot \mathrm{s}^{-1}$ ), umidade específica (colorido a cada 2 g.kg ${ }^{-1}$ ) e vento (vetores em m.s ${ }^{-1}$ ) para os casos: (a) PFSC (período de 10 a 17/05/2010) e (b) PFCC (período de 04 a 11/04/2010) sobre o ponto de grade da Reanálise ERA-Interim localizado em $22,5^{\circ} \mathrm{S} / 43,5^{\circ} \mathrm{W}$. O retângulo tracejado marca o período pós-frontal analisado.

com a altura também mostra a advecção fria desde os baixos até os altos níveis.

Em resumo, observa-se que o caso PFCC está associado a ventos de forte intensidade e de direção sul, que sopram perpendiculares à linha da costa. Tais ventos convergem na faixa litorânea, promovendo movimento ascendente. No caso PFSC, não ocorre convergência de vento no litoral, o movimento vertical é subsidente e advecção de temperatura é nula. Além disso, o posicionamento do AM ao sul da região em estudo e o ciclone sobre o oceano, ambos não muito intensos, promovem ventos fracos de componente oeste que mantém a estabilidade da atmosfera. Pode-se afirmar que a direção e intensidade do vento na região são fatores determinantes para a caracterização do evento PFCC ou PFSC, pois auxiliam no transporte de umidade, convergência e movimento ascendente junto à costa.

\section{Conclusões}

Neste artigo é feito um levantamento de todos os SFs que atingiram o ERJ no período entre 2000 e 2013. A motivação foi investigar um fenômeno denominado localmente de "chuva por efeito de circulação marítima", que até o momento não havia sido documentado. Quando da ocorrência desse fenômeno, os previsores observam que, apesar do SF posicionar-se ao norte da região em estudo sem afetar o MRJ, ocorre precipitação fraca a moderada sobre a cidade, de origem estratiforme, trazida pelos ventos que sopram do oceano em direção ao continente.

Com o objetivo de desenvolver uma climatologia de tais eventos e identificar as condições sinóticas associadas ao fenômeno, os casos pós-frontais foram classificados em três tipos: Pós-Frontais Sem Chuva (PFSC); Pós-Frontais Com Chuva (PFCC) e Pós-Frontais Excluídos (PFE), nos quais não é possível identificar a saída do SF da região em estudo. A partir dessa separação, foram construídos com- postos para os casos PFCC e PFSC usando dados da Reanálise ERA-Interim.

Os resultados da climatologia indicam que no período de 2000 a 2013 (14 anos), foram obtidos 473 passagens de SFs pelo MRJ, sendo: 150 PFSC, 75 PFCC e 248 PFE. Isso mostra que mais da metade dos SFs que atingem o ERJ (aproximadamente 52\%) não evoluem de forma clássica, ou seja, permanecem estacionários sobre a região em estudo ou deslocam-se para leste, sobre o oceano, impossibilitando a definição da situação pós-frontal. Dos casos restantes, $32 \%$ são do tipo PFSC e 16\% do tipo PFCC. Os casos PFCC são mais frequentes no outono, sendo os meses de maio e julho os de maior frequência de ocorrência (média de 0,9 sistema por mês). Durante todo o período estudado, não houve ocorrência de caso PFCC no mês de dezembro. Os casos PFSC predominam no inverno e primavera, com máximos nos meses de agosto e setembro (médias de 1,4 e 1,5 sistemas, respectivamente). Já os casos PFE são mais frequentes no verão. A elevada frequência de casos PFE motivou uma investigação mais detalhada de tais eventos, que será apresentada em um futuro artigo.

A análise de compostos mostrou que nos casos PFCC, o cavado sobre o oceano, na retaguarda do SF encontra-se mais amplificado e o AM encontra-se mais intensificado do que no composto PFSC. A convergência associada ao SF em PFCC também é mais intensa em toda a sua extensão, do que em PFSC. Ao sul do ERJ nos dois compostos os ventos sopram de sul e com giro anticiclônico, perpendiculares à linha da costa, provenientes do AM. Com relação ao movimento vertical, em ambos os compostos ocorre movimento ascendente sobre o ERJ, sendo mais intenso no composto PFCC.

Os estudos de casos possibilitaram uma análise mais detalhada de dois eventos selecionados, um ocorrido em maio de 2010 (PFSC) e outro em abril de 2010 (PFCC). A comparação entre os casos indicou que no caso PFCC havia maior conteúdo de umidade e ventos mais intensos, responsáveis por aumentar o fluxo de umidade integrado ver- 
ticalmente, além de maior teor de água precipitável e maior gradiente térmico.

Pode-se concluir que a precipitação pós-frontal, que ocorre no MRJ quando o SF já se encontra bem afastado da região, está associada à chegada de ventos de sul que aumentam a convergência na costa, gerando movimento ascendente e promovendo o levantamento da umidade da camada inferior da atmosfera. Esse ar relativamente frio e úmido é forçado a ascender e se condensa, formando nuvens estratiformes que precipitam de forma contínua sobre a cidade.

\section{Agradecimentos}

Este artigo foi desenvolvido como parte do trabalho de conclusão de curso da primeira autora, com apoio da Fundação de Amparo à Pesquisa do Estado do Rio de Janeiro (FAPERJ), através da Bolsa de Iniciação Científica PIBIC (Processo número E-26/101.692/2008) e do Conselho Nacional de Desenvolvimento Cientifico e Tecnológico (CNPq) através de Bolsa PIBIC/UFRJ.

\section{Referências}

BONNET, S.M. Caracterização sinótica e climatológica de eventos de chuva pós-frontal no Rio de Janeiro. Trabalho de conclusão de curso, IGEO, UFRJ, Rio de Janeiro, 2012.

CAVALCANTI, I.F.A.; KOUSKY, V. Frentes Frias sobre o Brasil. Tempo e Clima no Brasil, p. 135-145, 2009.

CHAVES, R.R.; CAVALCANTI, I.F.A. Atmospheric Circulation Features Associated with Rainfall Variability over Southern Northeast Brazil. Monthly Weather Review, v. 129, p. 2614-2626, 2001.

DEE, D.P.; UPPALA, S.M.; SIMMONS, A.J.; BERRISFORD, P.; POLI, P. et al. The ERA-Interim reanalysis: configuration and performance of the data assimilation system. Quarterly Journal of the Royal Meteorological Society, v. 137, p. 553-597, 2011.
DERECZYNSKI, C.P.; OLIVEIRA, J.S.; MACHADO, C.O. Climatologia da precipitação no município do Rio de Janeiro. Revista Brasileira de Meteorologia, v. 24, n. 1, p. 24-38, 2009.

DOMINGUES, I.P.; DERECZYNSKI, C.P. Estudo de caso de chuva no Rio de Janeiro por efeito de circulação marítima. In: IX Congresso Argentino de Meteorologia (IX CONGREMET). Anais... 2005.

GAN, M.A.; KOUSKY, V.E.; ROPELEWSKI, C.F. The South America monsoon circulation and its relationship to rainfall over West-Central Brazil. Journal of Climate, v. 17, p. 47-66, 2004.

LIMA, M.C. Variabilidade da Precipitação no Litoral Leste da Região Nordeste do Brasil. Dissertação de Mestrado em Meteorologia, INPE-5283-TDI/453, 1991.

MELLO, G.M.S.; DERECZYNSKI, C.P. Estudos de casos de precipitação no Rio de Janeiro provocados pela intensificação da brisa marítima. In: XV Congresso Brasileiro de Meteorologia. Anais... 2008.

MELLO, G.M.S. Estudo de caso de precipitação pós-frontal no município do Rio de Janeiro. Trabalho de conclusão de curso, IGEO, UFRJ, Rio de Janeiro, RJ, 2009.

NOBRE, C.A.; MATTOS, L.F.; DERECZYNSKI, C.P.; TARASOVA, T.A.; TROSNIKOV, I.V. Overview of atmospheric conditions during the Smoke, Clouds, and Radiation-Brazil (SCAR-B) field experiment. Journal of Geophysical Research, v. 103, n. D24, p. 31809-31820, 1998.

OLIVEIRA, A.S. Interações entre sistemas frontais na América do Sul e a conveç̧ão na Amazônia. 115 p. Dissertação de Mestrado - INPE/SJC, São José dos Campos - SP, 1986.

RICHTER, I.; MECHOSO, C.R.; ROBERTSON, A.W. What Determines the Position and Intensity of the South Atlantic Anticyclone in Austral Winter? - An AGCM Study. Journal of Climate, v. 21, p. 214-229, 2008.

SATYAMURTY, P.; NOBRE, C.A.; SILVA DIAS, P.L. Tropics - South America. In: Meteorology of the Southern Hemisphere. Eds. KAROLY, D.J.; VINCENT, D.G. American Meteorological Society, Meteorological Monographs, v. 27, n. 49, p. 119-139, 1998.

This is an Open Access article distributed under the terms of the Creative Commons Attribution Non-Commercial License which permits unrestricted non-commercial use, distribution, and reproduction in any medium provided the original work is properly cited. 